\title{
Elastic Constants and Pressure Derivatives of Calcium Oxide
}

\author{
Krishna Murti Raju \\ Department of Physics, Bipin Bihari Post Graduate College, Jhansi, India \\ Email: rajukm@yahoo.com
}

Received 6 August 2014; revised 26 September 2014; accepted 27 October 2014

Copyright (C) 2014 by author and OALib.

This work is licensed under the Creative Commons Attribution International License (CC BY). http://creativecommons.org/licenses/by/4.0/

(c) (i) Open Access

\begin{abstract}
The electrostatic and Börn-Mayer repulsive potentials method was used to calculate the higher order elastic constants and pressure derivatives of alkaline-earth oxide $\mathrm{CaO}$ in a wide temperature range $(100-1000 \mathrm{~K})$. The calculations have been made starting from primary physical parameters viz. nearest-neighbour distance and hardness parameter assuming long- and shortrange potentials. The different results of the calculations are compared with experimental data and discussed.
\end{abstract}

Keywords

Anharmonic Properties, Higher Order Elastic Constants, Pressure Derivatives, CaO

Subject Areas: Composite Material, Computational Chemistry, Condensed State Physics, Environmental Chemistry

\section{Introduction}

Nowadays, calculations for the anharmonic properties have reached a sufficient degree of sophistication to reproduce satisfactorily experimental data and to obtain interesting properties in the cases in which experimental measurements are fully lacking. Among these properties, elastic constants represent a good test for estimating the quality of a theoretical approach. As a matter of fact, they require computation, point by point, of the hypersurface of the total energy for applicable lattice deformations and numerical calculation of the second derivatives of the energy with respect to strain components. The electrostatic and Börn-Mayer repulsive potentials method [1] implemented in the crystal allows this type of calculation as the cyanides, chalcogenides, alkali halides, alkaline oxides and sulphides examples shown in [2]-[7]. A good agreement obtained for the temperature dependence of anharmonic properties, encouraged the author to extend this model for the study of the temperature dependence of higher order elastic constants and pressure derivatives for divalent materials. This study extends the previous one on $\mathrm{MgO}$ [6] to the alkaline-earth oxide $\mathrm{CaO}$ and can be motivated as follows: 1) The 
three oxides (MgO, $\mathrm{CaO}, \mathrm{SrO}$ ) have the same cubic rock-salt type structure (space group: $F m 3 m$ ) and represent a family of similar fully ionic compounds. 2) Very accurate experimental values of elastic constants are available and they are obtained by high accuracy techniques and can be considered as references to test the quality of theoretical approaches. The study of alkaline-earth oxides is of great interest for many other reasons. These oxides have long been considered a typical case for understanding bonding in ionic oxides and are also one of the most fundamental materials for industrial science. These oxides are a major constituent of the earth's lower mantle (between 600 and $2900 \mathrm{~km}$ in depth). Therefore studies on elastic, thermal and structural properties for such compounds have great importance.

\section{Formulation}

The elastic energy density for a crystal of a cubic symmetry can be expanded up to quartic terms as shown below:

$$
U_{0}=U_{2}+U_{3}+U_{4}=[1 / 2 !] C_{i j k l} x_{i j} x_{k l}+[1 / 3 !] C_{i j k l m n} x_{i j} x_{k l} x_{m n}+[1 / 4 !] C_{i j k l m n p q} x_{i j} x_{k l} x_{m n} x_{p q},
$$

where $C_{i j k l}, C_{i j k l m n}$ and $C_{i j k l m p q}$ are the SOECs, TOECs and FOECs in tensorial form; $x_{i j}$ are the Lagrangian strain components; $C_{\mathrm{IJ}}, C_{\mathrm{IJK}}$ and $C_{\mathrm{IJKL}}$ are the SOECs, TOECs and FOECs in Brügger's definition and Voigt notations. The SOECs, TOECs and FOECs are as given below:

$$
\begin{aligned}
& C_{i j k l}=C_{\mathrm{IJ}}=\left(\partial^{2} U / \partial x_{i j} \partial x_{k l}\right)_{x}=0, \\
& C_{i j k l m n}=C_{\mathrm{IJK}}=\left(\partial^{3} U / \partial x_{i j} \partial x_{m n} \partial x_{k l}\right)_{x}=0, \\
& C_{i j k l m n p q}=C_{\mathrm{IJKL}}=\left(\partial^{4} U / \partial x_{i j} \partial x_{k l} \partial x_{m n} \partial x_{p q}\right)_{x}=0 .
\end{aligned}
$$

An elastic constant consists of two parts as follows

$$
C_{\mathrm{IJ}}=C_{\mathrm{IJ}}^{0}+C_{\mathrm{IJ}}^{\mathrm{vib}} ; \quad C_{\mathrm{IJK}}=C_{\mathrm{IJK}}^{0}+C_{\mathrm{IJK}}^{\mathrm{vib}} ; \quad C_{\mathrm{IJKL}}=C_{\mathrm{IJKL}}^{0}+C_{\mathrm{IJKL}}^{\mathrm{vib}} .
$$

The first part is the strain derivative of the internal energy $U_{o}$ and is known as "static" elastic constant and the second part is the strain derivative of the vibrational free energy $U^{\mathrm{vib}}$ and is called "vibrational" elastic constant. The superscript “"”, has been introduced to emphasize that the static elastic constants correspond to $0 \mathrm{~K}$. By adding the vibrational elastic constants to the static elastic constants, one may get SOECs and TOECs at any temperature for $f c c$ crystals. The general expressions for these properties have been reported in [1] [5] and therefore are not repeated in this paper.

\section{Evaluation}

The brief introduction of formulation is given in the preceding Section 2. The whole evaluation is based on the assumption that the crystal structure of the material does not change when temperature varies up to their melting point. Using the concept of nearest-neighbour distance and hardness parameter, the elastic constants and pressure derivatives for $\mathrm{CaO}$ are evaluated at different temperatures (from $100-1000 \mathrm{~K}$ ) using the formulation and shown in Figures 1-7. The comparison has also been made for some elastic properties for $\mathrm{CaO}$ crystal with available theoretical and experimental data [8]-[10] and presented in Table 1.

\section{Results and Discussions}

In order to understand the thermodynamic and thermo-elastic behaviour of solids at high temperatures, it is necessary to have reliable values of elastic constants corresponding to such temperatures. The elasticity offers more information than the volume in interpreting the temperature dependence of equation of state because the compressibility is defined by the derivative of volume. The elastic constants also provide a ground for examining of earth's deep interior. Besides being of considerable theoretical interest there is still no complete general theory that should be used for precise calculation of elasticity. The problem becomes more difficult if we consider the case of minerals of geophysical interest [11]. Therefore we have to develop semi empirical and phenomenological model formulations for predicting the values of elastic constants for solids at higher temperatures. There have 


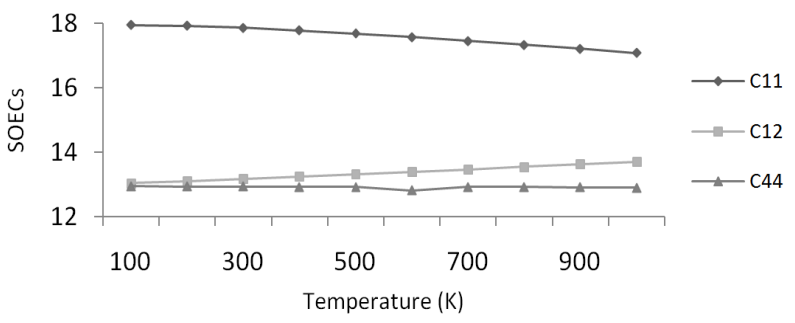

Figure 1. Temperature variation of SOECs $\left(10^{11} \mathrm{dyne} / \mathrm{cm}^{2}\right)$.

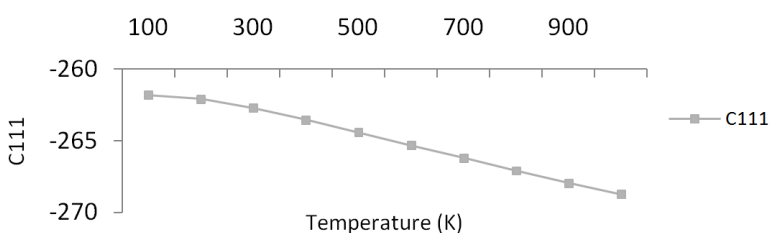

(a)

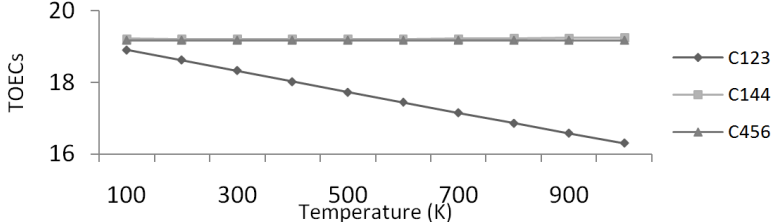

(b)

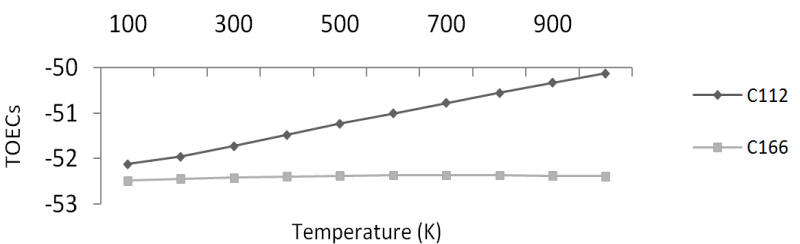

(c)

Figure 2. Temperature variation of TOECs $\left(10^{11} \mathrm{dyne} / \mathrm{cm}^{2}\right)$.

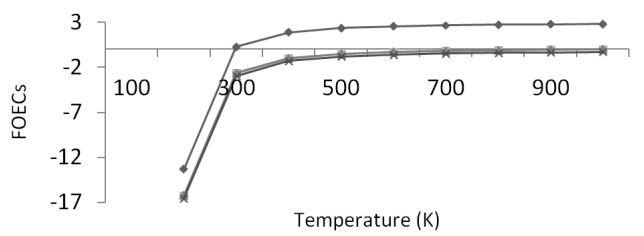

(a)

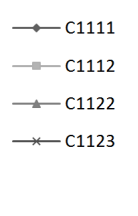

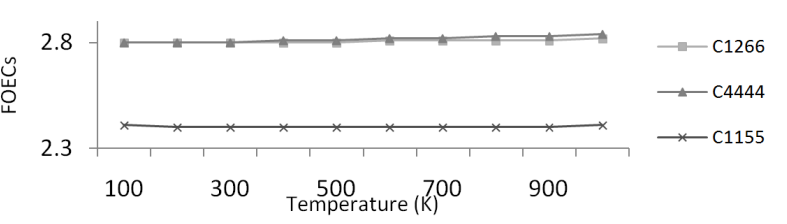

(b)

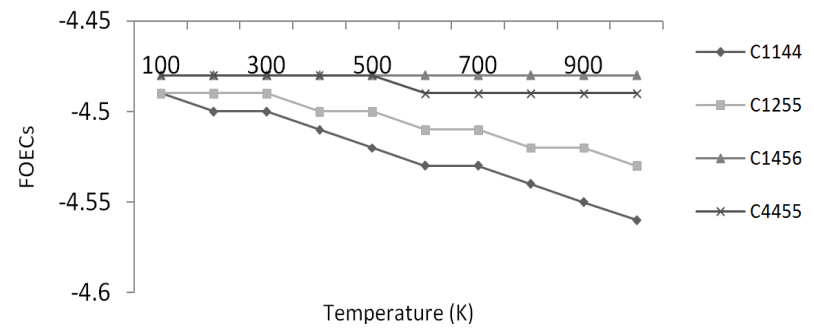

(c)

Figure 3. Temperature variation of FOECs. (3) In $10^{14} \mathrm{dyne} / \mathrm{cm}^{2}$; (b) In $10^{13} \mathrm{dyne} / \mathrm{cm}^{2}$; (c) In $10^{12}$ dyne/cm 2

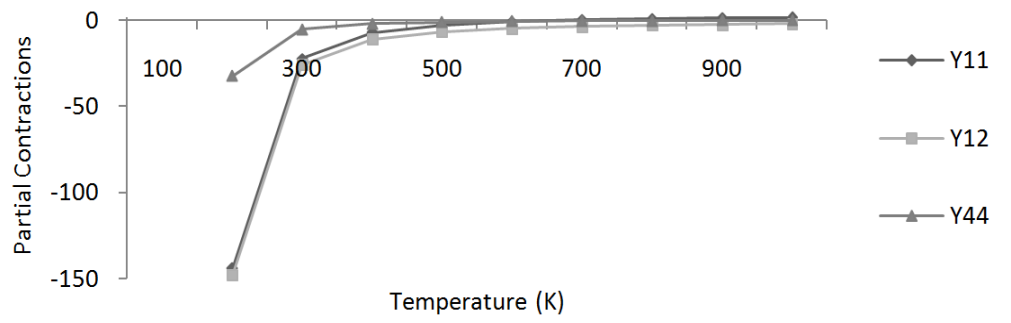

Figure 4. Temperature variation of Partial Contractions (in $10^{14}$ dyne/cm ${ }^{2}$ ). 


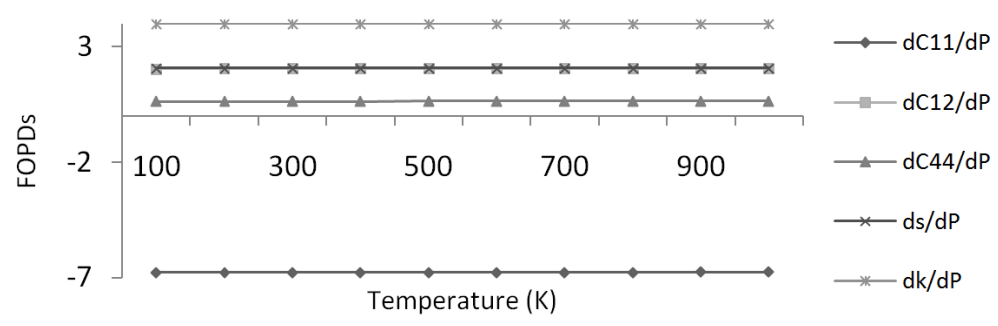

Figure 5. Temperature variation of FOPDs of SOECs.

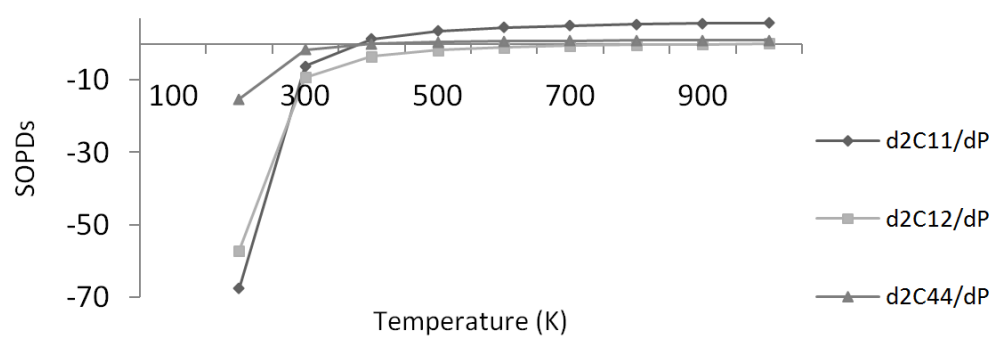

Figure 6. Temperature variation of SOPDs (in $10^{-11} \mathrm{dyne} / \mathrm{cm}^{2}$ ).

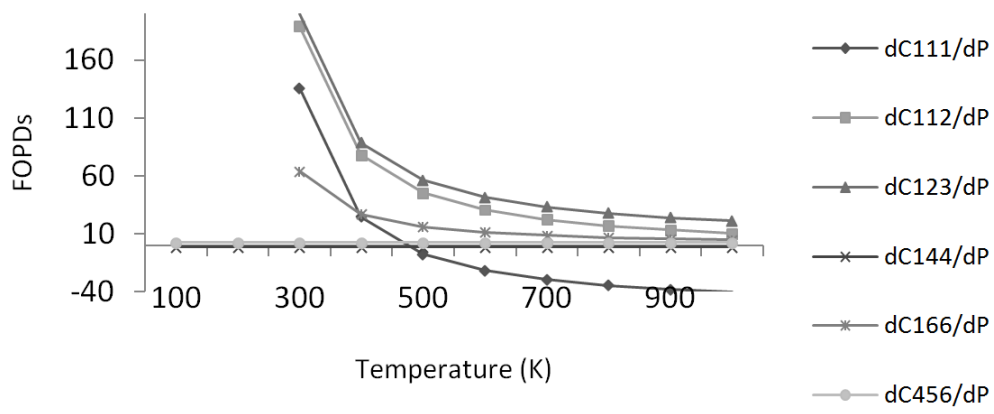

Figure 7. Temperature variation of FOPDs of TOECs.

Table 1. Comparison data of elastic properties (in $10^{10}$ Newton/meter ${ }^{2}$ ) for $\mathrm{CaO}$ at room temperature. Experimental values are given within bold parantheses (compiled from Ref. [8]-[10]).

\begin{tabular}{|c|c|c|c|c|c|c|c|c|c|}
\hline $\mathrm{C}_{111}$ & $\mathrm{C}_{112}$ & $\mathrm{C}_{123}$ & $\mathrm{C}_{144}$ & $\mathrm{C}_{166}$ & $\mathrm{C}_{456}$ & ds/dp & $\mathrm{dk} / \mathrm{dp}$ & $\mathrm{dC}_{44} / \mathrm{dp}$ & Ref. \\
\hline-26.27 & -5.17 & 1.83 & 1.92 & -5.24 & 1.91 & 2.08 & 4.01 & 0.64 & Present \\
\hline-30.23 & -0.76 & 0.95 & 1.21 & 2.39 & 1.34 & 3.4 & $3.26(\mathbf{6 . 0 )}$ & $0.42(\mathbf{0 . 6 )}$ & [8] \\
\hline-25.16 & -5.35 & 1.58 & 1.81 & -6.73 & 1.98 & $1.85(\mathbf{0 . 6 )}$ & 3.96 (3.4) & 1.23 & [9] \\
\hline-26.8 & -1.36 & 1.58 & 1.87 & -2.72 & 2.01 & 3.01 (4.1) & $3.18(4.8)$ & $0.17(\mathbf{0 . 2})$ & [10] \\
\hline
\end{tabular}

been continuous efforts to study the temperature dependence of elastic constants and related properties of ionic solids. In past years, the physical properties of $\mathrm{CaO}$ have been studied [12] [13]. However, none of the work reported in the literature so far is centred on the study of anharmonic properties of CaO. Thus, in the present work, we have studied the anharmonic properties of this alkaline-earth compound. The results obtained in this investigation can be used for further study of the material. Our whole theoretical approach can be applied to the evaluation of related parameters to study the anharmonic and micro-structural properties of CaO. The experimental data for elastic parameters for many solids have been reported in literature which is considered to be most precise. Here the elastic properties of $\mathrm{CaO}$ are computed. For $\mathrm{CaO}$ the value of melting temperature is $2853 \mathrm{~K}$.

For a cubic solid, there are three independent second order elastic constants viz. $\mathrm{C}_{11}, \mathrm{C}_{12}$ and $\mathrm{C}_{44}$. The temperature variation of SOECs for $\mathrm{CaO}$ is shown in Figure 1. The elastic constants of solids in general decrease with temperature and such a decrease has been explained by many available theories. But in the NaCl-like structure the elastic constant $\mathrm{C}_{12}$ of some alkali halides (for example $\mathrm{KCl}, \mathrm{KBr}$ etc.) is increasing with temperature. This phenomenon is known as the anomalous temperature dependence of $\mathrm{C}_{12}$ in these solids. In the present work, the 
temperature dependence of $\mathrm{C}_{12}$ is found to explain the observed anomalous temperature dependence of $\mathrm{C}_{12}$ in crystals with NaCl-structure. Results are presented for $\mathrm{CaO}$. We see that an anomalous temperature dependence of $\mathrm{C}_{12}$ does occur in this material. It is interesting to note from Figure 1 that the values of $\mathrm{C}_{11}$ is decreasing with increase in temperature, while the value of another elastic constant $\mathrm{C}_{44}$ is nearly constant with increase in temperature. On the other hand, the value of $\mathrm{C}_{12}$ is increasing with increase in temperature. The values of $\mathrm{C}_{44}$ are generally lower than the values of $\mathrm{C}_{12}$. The abnormal behavior of the temperature dependence of the elastic constant $\mathrm{C}_{12}$ is related to the existence of many body potential and non-central potentials in solids, which are responsible for the breakdown of the cauchy relation $\mathrm{C}_{12}=\mathrm{C}_{44}$. The variation of $\mathrm{C}_{11}$ with temperature is found to be large as compared with $\mathrm{C}_{12}$ and $\mathrm{C}_{44}$. The constant $\mathrm{C}_{11}$ represents elasticity in length. A longitudinal strain produces a change in volume without change in shape. The volume change is closely related to the temperature and thus produces a large change in $\mathrm{C}_{11}$. On the other hand, the constant $\mathrm{C}_{12}$ and $\mathrm{C}_{44}$ are related to the elasticity in shape which is a shear constant. A transverse strain or shearing causes a change in shape without a change in volume. Therefore, $\mathrm{C}_{12}$ and $\mathrm{C}_{44}$ are less sensitive to the temperature. Thus, study of the temperature dependence of $\mathrm{C}_{11}$ may provide a more critical test of the theory.

The higher order elastic constants are strongly related to other anharmonic properties; such as thermal expansion, thermo elastic constants and thermal conductivity. The knowledge of TOEC may provide further critical data for testing the machines for non-destructive-testing. Furthermore, we expected to obtain additional data for the discussion of the influence of asymmetric ions on non-linear elastic properties, in particular for crystals of rock salt type. Third order elastic constants play an important role in solid-state physics. They allow an evaluation of first order anharmonic terms of the inter-atomic potential or of generalized Grüneisen parameters, which enter the theories of all anharmonic phenomena, such as the interaction of acoustic and thermal phonons and the equation of state. The present study of the temperature variation of TOECs could prove useful in studies of various anharmonic properties of ionic solids in general. The TOECs play an important role when it comes to explain anharmonic phenomena in solids (interactions of ultrasonic vibration with thermal phonons, harmonic generators, equation of state etc.). As a result of the anharmonicity of the crystal lattice vibrations, the elastic constants vary with temperature. The Temperature variations of TOECs for $\mathrm{CaO}$ are shown in Figures 2(a)-(c). Among the calculated third order elastic constants of these materials, $\mathrm{C}_{111}$ 's are the largest in their absolute values and an order of magnitude larger than the SOEC. Magnitude of other $C_{i j k}$ 's are markedly smaller than those of $\mathrm{C}_{111}$. In Table 1, third order elastic constants are compared with available theoretical and experimental data. It is obvious from this Table that there is good agreement between the calculated values from this study and the previously reported values for $\mathrm{CaO}$. This close agreement shows the validity of present approach.

Third and fourth order elastic constants are required to study many anharmonic properties of crystals and therefore their accurate evaluation is essential. Recently attempts have been made to calculate anharmonic properties of various crystals. Only few of them are taken account the temperature dependence of these properties. The thermal contribution to elastic constants is very significant. The experimental data reveal that in going from $100 \mathrm{~K}$ to higher temperatures, the values of second order elastic constants are changed considerably even for highly ionic solids. We have already discussed the temperature variation of second and third order elastic constants of $\mathrm{CaO}$. Since the contribution from third and fourth order coupling parameters to many anharmonic properties are of the same order of magnitude, the knowledge of FOECs is equally important as that of TOECs. Calculated results of fourth order elastic constants at different temperatures are reported in Figures 3(a)-(c). Due to non-availability of experimental data, the comparison is not made. The Partial Contractions for $\mathrm{CaO}$ are shown in Figure 4.

Recent extension of ultrasonic techniques to high pressure and high frequencies renewed interest in the higher order coefficients of non-linear elasticity. Much theoretical work has been done on the temperature dependence of the elastic constants of materials. An investigation into the higher order elastic constants and their pressure derivatives provides useful information on the inter-atomic forces, inter-ionic potentials and on anharmonic properties of crystalline solids. This is why recently [14]-[17] there have been several attempts to determine the elastic constants of higher order using theoretical as well as experimental techniques. Calculated results of first and second order pressure derivatives of second order elastic constants at different temperatures are reported in Figure 5 and Figure 6. An important aspect of the present investigation is the calculation of second order pressure derivatives of SOECs at different temperatures (Figure 6). The FOPDs of the TOECs of $\mathrm{CaO}$ are presented in Figure 7. In Table 1, pressure derivatives are compared with available theoretical and experimental data. It is obvious from this Table that there is good agreement between the calculated values from this study and the previously reported values for $\mathrm{CaO}$. 
These data are valuable for the interpretation of the anomalous elastic behaviour of $\mathrm{CaO}$ and similar systems. The new data may offer an additional possibility to improve the theoretical models developed recently for the interpretation of the behaviour of elastic constants in elevated temperature segment. The present method can therefore be used to predict successfully the values of elastic constants for solids at higher temperatures. It is necessary to mention here that the linear relationship between elastic constants and temperature holds well only above the Debye temperature, i.e. in high temperature region. Thus, this study is valid from higher temperature (or room temperature) to the melting temperature for $\mathrm{CaO}$. The negative slops of the curves from Figures 1-7 reflect that decrement in the elastic constants is sharper than the increment in the temperature. It is appropriate to mention here that a similar variation is also observed for elastic properties by previous workers [18]-[20]. Therefore, we may say that the model suggested by Kailash $e t$ al. also holds well for other $f c c$ ionic materials, confirming the validity of generalisation method used by us. Thus, the results of the elastic properties of this alkaline-earth oxide computed with the help of the present theory are satisfactory and are comparable to the results obtained by various first-principle studies. Hence, the present theory may be useful for analyzing elastic behaviours under high temperature of other cubic structure solids. We have thus presented a simple method to study the elastic properties of solids under varying conditions of temperatures. The results obtained are encouraging. Due to the simplicity of the method, it can be applied to the more complicated solids, like minerals of geophysical importance and applications.

\section{References}

[1] Kailash, Raju, K.M., Shrivastava, S.K. and Kushwaha, K.S. (2007) Anharmonic Properties of Rocksalt Structure Solids. Physica B, 390, 270-280. http://dx.doi.org/10.1016/j.physb.2006.08.024

[2] Singh, R.K., Singh, R.P., Singh, M.P. and Chaurasia, S.K. (2008) Theoretical Analysis of Acoustic Attenuation and Nonlinearity in Barium Monochalcogenides in b2 Phase. Turkish Journal of Physics, 32, 211-218.

[3] Singh, D., Pandey, D.K. and Yadawa, P.K. (2009) Ultrasonic Wave Propagation in Rare-Earth Monochalcogenides. Central European Journal of Physics, 7, 198-205. http://dx.doi.org/10.2478/s11534-008-0130-1

[4] Kor, S.K., Singh, D. and Srivastava, A.K. (2005) Ultrasonic Studies of Thulium Monochalcogenides. Indian Journal of Pure and Applied Physics, 43, 355-358.

[5] Kailash (1996) Anharmonic Properties of Alkali Halides and Cyanides. Acta Physica Polonica A, 89, 75.

[6] Raju, K.M., Srivastava, R.K. and Kailash (2007) Temperature Variation of Higher Order Elastic Constants of MgO. Pramana, 69, 445-450. http://dx.doi.org/10.1007/s12043-007-0145-y

[7] Kailash and Raju, K.M. (2006) Theoretical Evaluation of Higher Order Elastic Constants and Pressure Derivatives of Some Magnesium Compounds. Indian Journal of Theoretical Physics, 54, 237.

[8] Garg, V.K., Puri, D.S. and Verma, M.P. (1977) On the Third-Order Elastic Constants of Ionic Solids with NaCl Structure. Physica Status Solidi (B), 80, 63-72. http://dx.doi.org/10.1002/pssb.2220800107

[9] Singh, R.P. and Shankar, J. (1979) Evaluation of Third-Order Elastic Constants and Pressure Dependence of SecondOrder Elastic Constants of Ionic Crystals. Physica Status Solidi (B), 93, 373-380. http://dx.doi.org/10.1002/pssb.2220930143

[10] Shankar, J. and Singh, J.P. (1982) Third- and Fourth-Order Elastic Constants for Silver Halides, Alkaline Earth Oxides, and Chalcogenides of Pb and Sn. Physica Status Solidi (A), 70, 677. http://dx.doi.org/10.1002/pssa.2210700237

[11] Srivastava, S.K. (2007) Relationship between Elastic Constants and Thermal Expansivity for Ionic Solids. Physica B, 387, 396-399. http://dx.doi.org/10.1016/j.physb.2006.04.025

[12] Lichanot, A. (2000) Hartree-Fock and Density Functional Calculations of the Elastic Constants of the Alkaline-Earth Oxides: Comparison with Experiment. Solid State Communications, 116, 543-546. http://dx.doi.org/10.1016/S0038-1098(00)00375-6

[13] Vijay, A. and Verma, T.S. (2000) Analysis of Temperature Dependence of Elastic Constants and Bulk Modulus for Ionic Solids. Physica B, 291, 373-378. http://dx.doi.org/10.1016/S0921-4526(00)00449-X

[14] Gibkes, J., Bein, B.K., Pelzl, J., Wieck, A., Frenzel, J., Eggeler, G., Holtfort, I. and Chirtoc, M. (2008) Processing Induced Change of Thermal Transport Properties of NITI-Shape Memory Alloy. Acta Physica Polonica A, 114, A97A101.

[15] Raju, K.M., Srivastava, R.K., Kumar, A. and Devi, S. (2010) Acoustical Investigation of Lead Chalcogenides. Physica B, 405, 4855-4857. http://dx.doi.org/10.1016/j.physb.2010.09.018

[16] Glorieux, C., Rostyne, K.V., Nelson, K., Gao, W., Lauriks, W. and Thoen, J. (2001) On the Character of Acoustic 
Waves at the Interface between Hard and Soft Solids and Liquids. Journal of the Acoustical Society of America, 110, 1299. http://dx.doi.org/10.1121/1.1396333

[17] Raju, K.M. (2011) Anharmonic Properties of Potassium Halide Crystals. Turkish Journal of Physics, 35, 323-340.

[18] Singh, P. and Gaur, N.K. (2014) Thermal and Elastic Properties of C60 in Fcc Phase. SOP Transactions on Theoretical Physics, 1, 68-72. http://dx.doi.org/10.15764/TPHY.2014.02006

[19] Raju, K.M. (2012) Effect of Temperature on Non-Destructive Wave Propagation in Potassium Halides. Physica B, 407, 3463-3466. http://dx.doi.org/10.1016/j.physb.2012.05.002

[20] Yana, N., Srivastava, S., Gupta, A.K. and Srivastava, Y. (2014) Study of Elastic and Acoustic Properties of TiN. Open Journal of Modern Physics, 1, 24-28. 